\title{
A general approach to combine the advantages of collinear and non-collinear spectrometer designs in phase-resolved second order nonlinear spectroscopy
}

\author{
Tobias Garling, ${ }^{\dagger}$ R. Kramer Campen, ${ }^{\ddagger} \dagger$ Martin Wolf, ${ }^{\dagger}$ and Martin Thämer, ${ }^{*} \dagger$ \\ $\dagger$ Department of Physical Chemistry, Fritz Haber Institute of the Max Planck Society, \\ Berlin, Germany \\ $\ddagger$ Faculty of Physics, University of Duisburg-Essen, Lotharstraße 1, 47048 Duisburg, \\ Germany \\ E-mail: thaemer@fhi-berlin.mpg.de
}

\section{Abstract}

Recent years have seen a huge progress in the development of phase sensitive second order laser spectroscopy which has proven to be a very powerful tool for the investigation of interfaces. These interferometric techniques involve the nonlinear interaction between three short laser pulses with the sample. In order to obtain accurate phase information, the relative phases between the pulses must be stabilized and their timings precisely controlled. Despite much progress made, fulfilling both requirements remains a formidable experimental challenge. The two common approaches employ different beam geometries which each yields its particular advantages and deficiencies. While non-collinear spectrometers allow for a relatively simple timing control they typically yield poor phase stability and require a challenging alignment. Collinear approaches in contrast come with a simplified alignment and improved phase stability but typically suffer from a highly limited timing control. In this contribution we present a general experimental solution which allows for combining the advantages of both approaches while being compatible with most of the common spectrometer types. Based on a collinear geometry we exploit different selected polarization states of the light pulses in welldefined places in the spectrometer to achieve a precise timing control. The combination of this technique with a balanced detection scheme allows for the acquisition of highly accurate phase resolved nonlinear spectra without any loss in experimental flexibility. In fact, we show that the implementation of this technique allows us to employ advanced pulse timing schemes inside the spectrometer, which can be used to suppress nonlinear background signals and extend the capabilities of our spectrometer to measure phase resolved sum frequency spectra of interfaces in a liquid cell.

\section{Introduction}

Second order nonlinear spectroscopies like sum frequency, difference frequency or second harmonic generation spectroscopy (SFG, DFG or SHG respectively) have recently become an indispensable tool for the characterization of interfaces. Compared to their linear counterparts these techniques offer the advantage of interface specificity (within the electric dipole approximation) provided the adjoining bulk media possess centrosymmetry. This intrinsic interface sensitivity opens up the possibility to 
selectively probe and characterize interfacial species and to study interfaces between bulk condensed phases. As a result of these unique properties SFG, DFG and SHG spectroscopies have been successfully applied to study interfacial phenomena in research areas, including medicine,,$^{1,2}$ electrochemistry, ${ }^{3}$, environmental ${ }^{4}$ and material sciences. ${ }^{5,6}$

In second order nonlinear spectroscopies two incident laser fields $E_{1}$ and $E_{2}$ with different (SFG, DFG) or equal frequencies (SHG) are overlapped at the desired interface to generate a third field $E_{s i g}$ which is be detected and analyzed. This nonlinear process is driven by the complex material response which is described by the second order susceptibility $\chi^{(2)} \cdot{ }^{7-9}$

$$
E_{S i g} \propto \chi^{(2)} E_{1} E_{2}
$$

The spectrum of $\chi^{(2)}$ reveals resonant transitions in the interfacial species at the pump and/or the signal frequencies, and the sign of its imaginary part $(+$ or -$)$ is directly related to their orientation with respect to the interface. ${ }^{7,10} \mathrm{In}$ order to extract the complex $\chi^{(2)}$ spectrum in a measurement the amplitude and phase of the generated signal field $E_{S i g}$ must be determined. Phase-sensitive techniques typically do so by superimposing $E_{S i g}$ with a reference beam of known phase, the so called local oscillator (LO). ${ }^{7,11}$ The detected heterodyned intensity $I_{d e t}$ is then given by

$$
I_{\text {det }}=\left|E_{\text {sig }}+E_{L O}\right|^{2}
$$

In order to obtain the desired phase and amplitude of the signal the quantity $E_{S i g}$ must be extracted from the measured intensity $I_{\text {det }}$. Various types of phase sensitive nonlinear spectrometers that have recently been designed follow different approaches to achieve this. They can roughly be divided into two types: ${ }^{11}$ scanning time domain approaches (narrowband or broadband versions) and broadband multiplexed frequency domain techniques. ${ }^{10,12-16}$ In time domain approaches the intensity modulation in $I_{d e t}$ is recorded while the relative phases of $E_{S i g}$ and $E_{L O}$ are shifted. This can be done by scanning the relative time delay between the pulses $E_{S i g}$ and $E_{L O}{ }^{12}$ or by modulating the carrier envelope phase within one of the pulses. This second possibility is exploited in a recently developed broad band time domain SFG scheme. ${ }^{13,17}$

In the alternative broadband frequency domain approach, the local oscillator pulse is typically delayed by a few picoseconds with respect to the signal pulse leading to sharp interference fringes in the spectral domain which can be recorded by a polychromator. Through inverse Fourier filtering of $I_{\text {det }}(\omega)$ phase and amplitude of $E_{\text {Sig }}$ can then be determined. ${ }^{15,18,19}$

Apart from the aforementioned differences all techniques rely on one common technical requirement: fine control over the relative phases between $E_{S i g}$ and $E_{L O}$ either for scanning the phases or for setting the pulse delays to the required values. ${ }^{11,18,19}$ The experimental implementation of such a control is, as we show in the following, nontrivial.

The advent of laser systems delivering femtosecond pulses has led to the development of broadband spectroscopic techniques which offer crucial advantages over the ordinary narrowband approaches. Performing nonlinear spectroscopy with such pulses increases the conversion efficiency of nonlinear processes and allows for covering a large spectral range in a single experiment without the need for time consuming frequency scans. ${ }^{20}$ Obviously, if the two pump pulses are short (fs to ps) they must be precisely overlapped in time to generate a nonlinear signal. In heterodyned second order spectroscopy both the LO and the sample signal are typically generated consecutively by the same pump pulse pair (or a replica extracted by a beamsplitter) to ensure a well defined phase relationship between $E_{S i g}$ and $E_{L O} \cdot{ }^{12,14,18,19,21}$ The pump pulses must then be temporally overlapped at both the sample and the location of LO generation (typically a reference crystal) in the spectrometer. As shown above it is simultaneously required that the relative time delay between the LO and the signal can be freely tuned. 


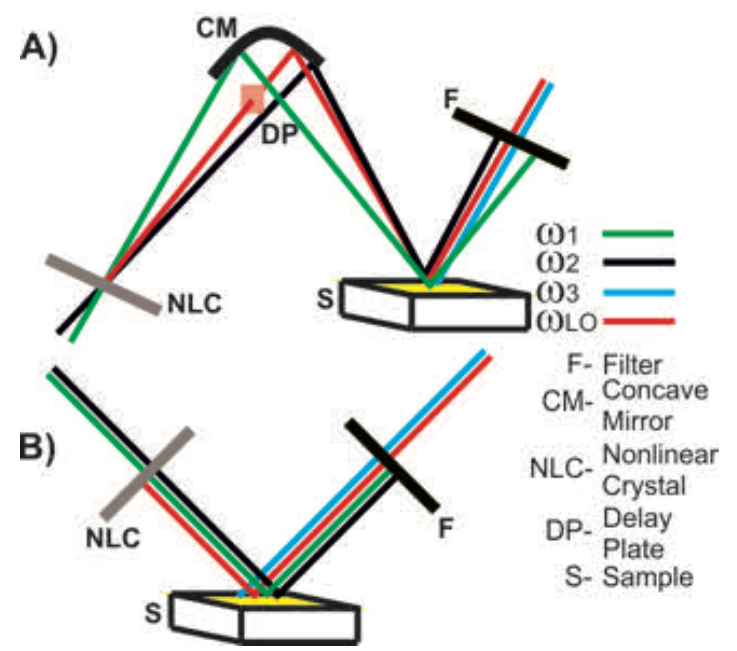

Figure 1: Common Heterodyned SFG spectroscopy setups. A) Non collinear geometry. B) Collinear geometry.

The most common solution to achieve this timing control is to use a non-collinear spectrometer design (see figure $1 \mathrm{~A}$ ). ${ }^{7,10,16,22}$ The pump pulses have thereby different incidence angles and the generated LO and signal waves are emitted in a third direction (determined through phase matching ${ }^{9}$ ). That way en route towards the sample the two pump pulses and the local oscillator all are spatially separated allowing for independent control of their phases (e.g. by introducing dispersive media of various thickness in any of the beams). Though the ease of timing control in this non-collinear approach is highly beneficial it comes with important technical and operational disadvantages. The spacial separation of the three beams between the reference and the sample position strongly reduces the phase stability of the interferometer and the resulting phase drifts can severely distort the obtained complex spectra. ${ }^{19}$ This phase instability can be reduced by using common optics for all beams (see figure $1 \mathrm{~A}$ ), which in turn leads to a strong reduction in degrees of freedom for the alignment of the three beams onto the sample. In particular obtaining a perfect spatial overlap of the incident pump beams at both the sample position and the reference crystal while simultaneously matching the angle of the emitted signal to the reflection angle of the LO now becomes a considerable experimen- tal obstacle. Furthermore, the phase matching direction or emission angle respectively for the signal and the LO depends in a non-collinear set up on the frequencies of the incident pulses ${ }^{9}$ making a repetition of the alignment procedure mandatory whenever one of the frequencies is modified. Finally, this approach causes the relative phase of the LO and the signal to be sensitive to the exact positioning of the sample which lowers comparability of the results from different samples.

All these challenges can be circumvented by using a full collinear design (figure $1 \mathrm{~B}$ ) which offers improved phase stability, largely simplified alignment and high phase reproducibility upon exchange of samples. ${ }^{15,23,24}$ However this comes at the price of reduced flexibility to control the pulse timings. Any dispersive material that is inserted into the beam path between the reference crystal and the sample position now acts on all the three beams simultaneously. Achieving a correct setting of the different pulse timings is hardly possible with this method. A second undesired side effect of the collinear geometry is the possible generation of background nonlinear signal contributions originating from the surfaces of the beam guiding optics inside the spectrometer. Due to the collinearity, the pump beams do not exclusively overlap at the sample and reference crystal position respectively but also at several other optical elements. Without a suitable experimental solution to this problem the resulting background contribution may be indistinguishable from the sample signal and can lead to distortion in the obtained complex spectrum. The comparison between the two spectrometer geometries seems to suggest that the choice to make use of the strength of one approach intrinsically excludes the possibility to benefit from the strengths of the other.

In this article we present a method that allows for combining the advantages of the noncollinear and the collinear spectrometer designs. The method is based on a collinear approach and we will show how a precise timing control of the involved beams can still be achieved without any loss in experimental flexibility. 
Furthermore, we will demonstrate how the contributions from background signals which appear in collinear spectrometers can efficiently be suppressed.

In the next section we first present the general principles and equations for the collinear timing control, followed by the result section, where we demonstrate the performance of the technique using two representative applications: the successful suppression of background signals and the measurement of the heterodyned vibrational second order spectrum of a self assembled monolayer inside an electrochemical cell.

\section{Concept}

The key to a precise timing control between the pulses $E_{S i g}$ and $E_{L O}$ in a collinear beam geometry without affecting the phases of the pump pulses $E_{1}$ and $E_{2}$ is to introduce the relative time delay between the signal and the LO in the detection beam path after the sample and the reference crystal. Here the presence of the pump pulses is not required, they can thus be filtered out, and only two beams with the same frequencies, the LO and the SFG signal remain propagating collinearly. To address the two pulses independently they need to differ in at least one property. One possible way is to make use of their respective polarization direction. Supposed $E_{S i g}$ and $E_{L O}$ have orthogonal polarization, then we can shift their timing using a birefringent crystal ${ }^{25}$ as shown in the following.

The time $T$ any light pulse takes to travel through a bulk material depends on its frequency $\omega$ and the corresponding refractive group index $n_{g}(\omega)$ as well as the distance $l$ :

$$
T=\frac{n_{g}(\omega) l}{c}
$$

with $c$ being the speed of light in vacuum. The distance $l$ depends on the bulk thickness $d$, the wave's angle of refraction $\alpha^{\prime}$ and its polarization. In the case of a uniaxial birefringent crystal two values for $l$ are obtained corresponding

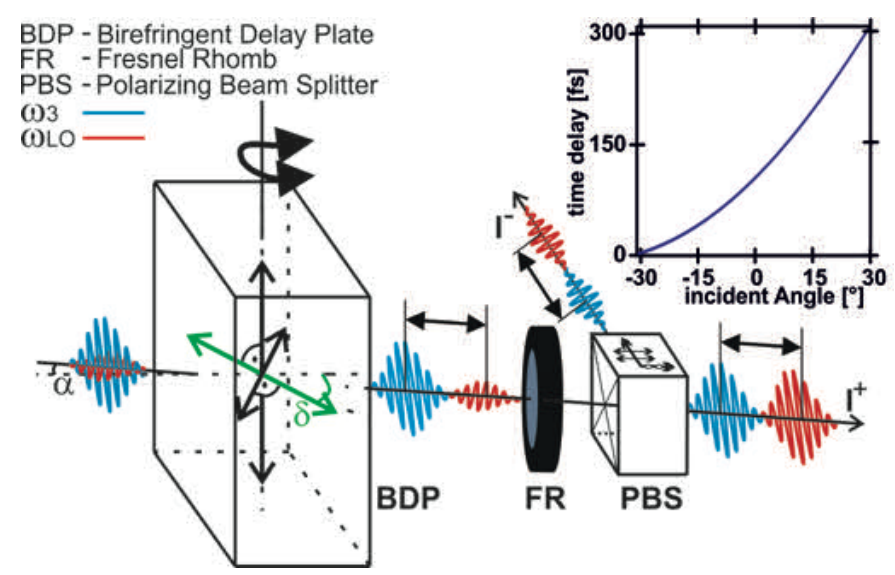

Figure 2: Working principle of the time delay compensation unit. The orthogonally polarized LO and Signal pulses (red and blue respectively) of same frequency pass a birefringent crystal which introduces a variable time delay between the pulses which can be varied as a function of the incidence angle $\alpha . \delta$ represents the angle between the surface normal and the optical axis (green). The Fresnel rhomb turns both beams' polarizations by $45^{\circ}$ and the polarizing beamsplitter projects the polarizations onto a horizontal and a vertical axes to achieve balanced detection. ${ }^{13,17}$ The so introduced time delay as a function of incidence angle $\left(d_{\text {Calcite }}=1 \mathrm{~mm}, \delta=25^{\circ}, \lambda_{S F G}=645 \mathrm{~nm}\right)$ is shown in the graph at the upper right corner.

to the ordinary and extraordinary propagation inside the crystal and is given by

$$
l_{o, e}=\frac{d}{\cos \left(\alpha_{o, e}^{\prime}+\rho_{o, e}\right)}
$$

Here $\rho$ is the walk-off angle which accounts for the mismatch between the Poynting and wave vector in anisotropic media. While $\rho$ is zero for the ordinary wave its value for the extraordinary wave can be calculated according to ${ }^{26}$

$$
\tan (\rho)=\frac{\sin \left(\delta+\alpha_{e}^{\prime}\right) \cos \left(\delta+\alpha_{e}^{\prime}\right)\left(n_{p, o}^{2}-n_{p, e}^{2}\right)}{n_{p, o}^{2} \sin ^{2}\left(\delta+\alpha_{e}^{\prime}\right)+n_{p, e}^{2} \cos ^{2}\left(\delta+\alpha_{e}^{\prime}\right)}
$$

In this equation $n_{p, o}$ and $n_{p, e}$ are the ordinary 
and extraordinary phase refractive indices respectively and $\delta$ the crystal cut angle as defined in figure 2 .

$\alpha^{\prime}$ is obtained via Snell's law as a function of the incident angle $\alpha$ (equation 6).

$$
\left(\alpha_{e, o}^{\prime}\right)=\sin ^{(-1)}\left(\frac{\sin (\alpha)}{n_{p ; e, o}(\omega)}\right)
$$

A pulse whose electric field is orthogonal to the optical axis (OA), such as the nonlinear signal, always experiences the same phase refractive index $n_{p, o}$ which is independent of the refracted angle. In contrast, a pulse whose electric field lies in the plane defined by the pulse's wave vector and the $\mathrm{OA}($ e.g. the local oscillator) will experience an effective phase refractive index $n_{p, e f f}$ given by ${ }^{27}$

$$
n_{p, e f f}=\frac{n_{p, o} n_{p, e}}{\sqrt{n_{p, o}^{2} \sin ^{2}\left(\delta+\alpha_{e}^{\prime}\right)+n_{p, e}^{2} \cos ^{2}\left(\delta+\alpha_{e}^{\prime}\right)}}
$$

Combining equations 6 and 7 the effective phase index can be calculated as a function of the incidence angle $\alpha$. For $\delta \neq 0$ the equations need to be solved numerically.

Inserting the result into equation 6 we obtain the refractive angle $\alpha^{\prime}$ which can be used to determine the individual transit times for the two pulses through the material (equations 3 and 4). The effective group index required for this calculation is the material constant $n_{g, o}$ for the ordinary wave while the corresponding value for the extraordinary wave can be calculated in the same manner as the phase index, using the respective values for the ordinary and extraordinary group refractive indices $n_{g, o}$ and $n_{g, e} \cdot{ }^{27}$

$$
n_{g, e f f}=\frac{n_{g, o} n_{g, e}}{\sqrt{n_{g, o}^{2} \sin ^{2}\left(\delta+\alpha_{e}^{\prime}\right)+n_{g, e}^{2} \cos ^{2}\left(\delta+\alpha_{e}^{\prime}\right)}}
$$

In order to obtain the overall relative delay of the two pulses upon arrival at the detector one has to finally account for the additional distance that the ordinary beam travels outside the birefringent crystal (equation 9) caused by the difference in the refracted angles between the ordinary and the extraordinary waves. ${ }^{27}$

$$
T_{e x}=\frac{1}{c}\left(\left(\tan \left(\alpha_{e}^{\prime}+\rho\right)-\tan \left(\alpha_{o}^{\prime}\right)\right) d \sin \alpha\right)
$$

The curve showing the overall induced time delay between the two pulses as a function of incident angle is also shown in figure 2 . The calculation was performed assuming a $1 \mathrm{~mm}$ thick calcite crystal with a crystal cut angle of $25^{\circ}$ at a wavelength of $645 \mathrm{~nm}$ which corresponds to our experimental parameters. The results show that such a birefringent crystal can indeed be used to smoothly vary the relative time delay between the LO and the nonlinear signal pulse over hundreds of femtoseconds. The crystal must be aligned such that the LO electric field lies either in or orthogonal to the plane defined by the wave vector and the OA. Larger delays if desired can be obtained using a thicker crystal or by modifying the crystal cut angle.

With this possibility of controlling the time delay between the signal and the LO pulse in hand we come to the question of how the interference between the two pulses can be detected. Within our assumption the two beams are orthogonally polarized and hence do not show any interference effects if detected directly. However they can be brought to interference by introducing a balanced detection scheme. ${ }^{13,17,28}$ To do so the polarizations of the two beams are each projected onto two new polarization axes which are rotated by $45^{\circ}$. This can be realized with a combination of a waveplate with a polarizing beamsplitter which are placed behind the birefringent crystal (see figure 2). The resulting two beams now both contain the interference between the two pulses, however, with opposite signs as shown in equation 10 and depicted in figure $2 .{ }^{13}$ 


$$
\begin{aligned}
I^{-} & =\frac{1}{2}\left|E_{S i g}\right|^{2}+\frac{1}{2}\left|E_{L O}\right|^{2}-E_{S i g} E_{L O} \\
I^{+} & =\frac{1}{2}\left|E_{S i g}\right|^{2}+\frac{1}{2}\left|E_{L O}\right|^{2}+E_{S i g} E_{L O}
\end{aligned}
$$

Recording the difference of the detected intensities from both resulting beams allows to isolate the pure interference term. Furthermore the cancellation of the square terms $\left|E_{\text {Sig }}\right|^{2}$ and $\left|E_{L O}\right|^{2}$ in the difference of the detector response efficiently reduces noise that originates from intensity fluctuations, mainly from the much stronger LO. Improvements of one order of magnitude in signal to noise ratio can easily be achieved with this detection scheme. ${ }^{17}$

So far, we have assumed that the signal and LO waves are orthogonally polarized. However, this is not a mandatory requirement for this detection scheme. The described optical arrangement rather acts similar to a polarization filter to the signal wave. Any polarization component parallel to the polarization of the local oscillator is eliminated by applying the difference in this balanced detection scheme since parallel components lead to interference terms with equal signs for both detectors. The polarization direction of the LO hence defines the detected polarization direction of the signal. To switch the detection from one polarization direction of the signal to another, it is sufficient to simply change the polarization of the LO. Note, because this leads to an interchange of polarization directions between the signal and LO also the orientation of the birefringent crystal needs to be changed by rotation about its surface normal and readjusting the tilt angle. In conclusion, by placing the birefringent crystal and the balanced detection scheme in series in the detection beam path we can obtain control over the relative pulse delays and detect any polarization component of a nonlinear signal from the sample. Importantly this holds independently from the polarization states of the pump pulses since they are no longer present in the detection beam path.

One additional important requirement for this type of detection is, however, that the LO ex- hibits a clean linear polarization. Furthermore this polarization must be tunable to any polarization direction independent of the selected polarizations of the pump beams. Achieving this in a collinear beam geometry is again nontrivial because in this case the polarization of the LO cannot be controlled by placing a waveplate and a polarizer into the LO beam. One solution is to use a thin z-cut alpha quartz crystal for the generation of the LO, which is placed at normal incidence into the beam path of the two collinear pump pulses before or after the sample. As a result of the non-centrosymmetric crystal structure of the alpha quartz, a second order signal is generated inside the bulk which is emitted in transmission direction and can be subsequently used as LO. Since in our collinear case all beams travel along the quartz' OA the pulses do not experience any birefringence and as a consequence the resulting LO pulse is perfectly linearly polarized. Furthermore as shown in the SI the polarization direction of the LO can be continuously tuned by simply rotating the quartz crystal about its z-axis. This property thereby holds for any combination of pump beam polarization. The use of the z-cut quartz wafer to generate the LO therefor offers precisely that degree of flexibility in polarization control which is needed.

As illustrated above the technical solution to include all the advantages of a non-collinear beam geometry in a collinear spectrometer design contains three parts: (1) The control of the relative time delay between the LO and the signal using a birefringent crystal, (2) the balanced detection scheme to extract the interference term and (3) LO generation with polarization control in a z-cut alpha-quartz crystal. There have recently been quite similar approaches published where pulse delay control is achieved by exploiting the properties of a birefringent crystal inside the spectrometer (mainly for SHG spectrometers). ${ }^{29}$ However, the way this timing control is implemented requires a certain polarization combination between pump and signal pulses which limits the applicability of the technique to particular experimental settings. In contrast, the approach 


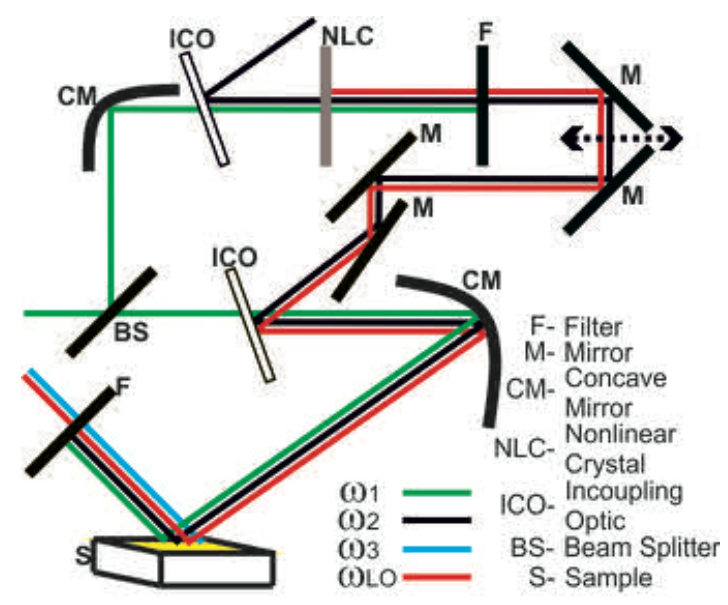

Figure 3: Schematic representation of our phase sensitive collinear time domain SFG spectrometer.

presented here is a general solution which is compatible with most of the common SHG and SFG spectrometer types (time domain as well as frequency domain techniques) without imposing any limitations on the choice of beam polarizations. Furthermore it can be implemented in already existing homodyned second order spectrometers quite easily to upgrade them into more powerful, collinear, phase sensitive heterodyned versions.

\section{Results and discussion}

After the introduction of the concept for adjusting the delay between the local oscillator (LO) and the nonlinear sample response in collinear spectrometers we now demonstrate its experimental application. The experiments are carried out using our recently developed phase sensitive, collinear, time domain SFG spectrometer shown in figure 3 where we implemented the described optical set up in the detector beam path. ${ }^{17}$ In this time domain approach the sum frequency signal and the LO pulse are generated by nonlinear mixing of two ultrashort pump pulses, one in the infrared spectral range and the other centered at $800 \mathrm{~nm}$. The 800 $\mathrm{nm}$ beam is aligned to consecutively pass a $\mathrm{z}$ cut quartz wafer at which the LO is generated and the sample while the beam is overlapped at these two positions with individual portions of the infrared beam in a collinear fashion (see figure 3). The separation of the two IR portions is achieved by the use of a beamsplitter and both partial beams are fed into the $800 \mathrm{~nm}$ beam path by two custom-made incoupling optics. After filtering out the pump beams the resulting heterodyned SFG signal passes a calcite plate for pulse delay control to be finally detected using the aforementioned balanced detection setup. An interferogram is recorded by modulating the carrier envelope phase (CEP) and amplitude of the SFG signal with respect to the CEP of the LO while the SFG signal and the LO temporally overlap to maximize the interference amplitude. ${ }^{13}$ The CEP modulation is obtained by shifting the relative phases (changing the time delays) of the two infrared portions used to generate the LO and signal pulse respectively. Fourier transformation of the resulting interferogram yields the complex valued SFG spectrum which is frequency resolved along the IR spectral axes. More details about the optical setup can be found in the supporting information and in the corresponding publications. ${ }^{17,21}$

\section{Suppression of background SFG contributions}

In a first example to demonstrate the capabilities of our technique we turn to the problem of possible contamination of a nonlinear signal with background contributions caused by the collinear design (see introduction). In our spectrometer background signals can originate from the incoupling optic and the focusing parabolic mirror depicted in figure 3. This situation is schematically shown in figure $4 \mathrm{~A}$. Although these background contributions are generally small because the beams are usually not focused other than onto the sample and the reference crystal for the generation of the LO they can become significant when samples are investigated which only yield small nonlinear signals.

Figure 4A shows a measurement of the nonresonant SFG response of a z-cut alpha quartz sample which represents this case. The obtained interferogram contains the sample signal 


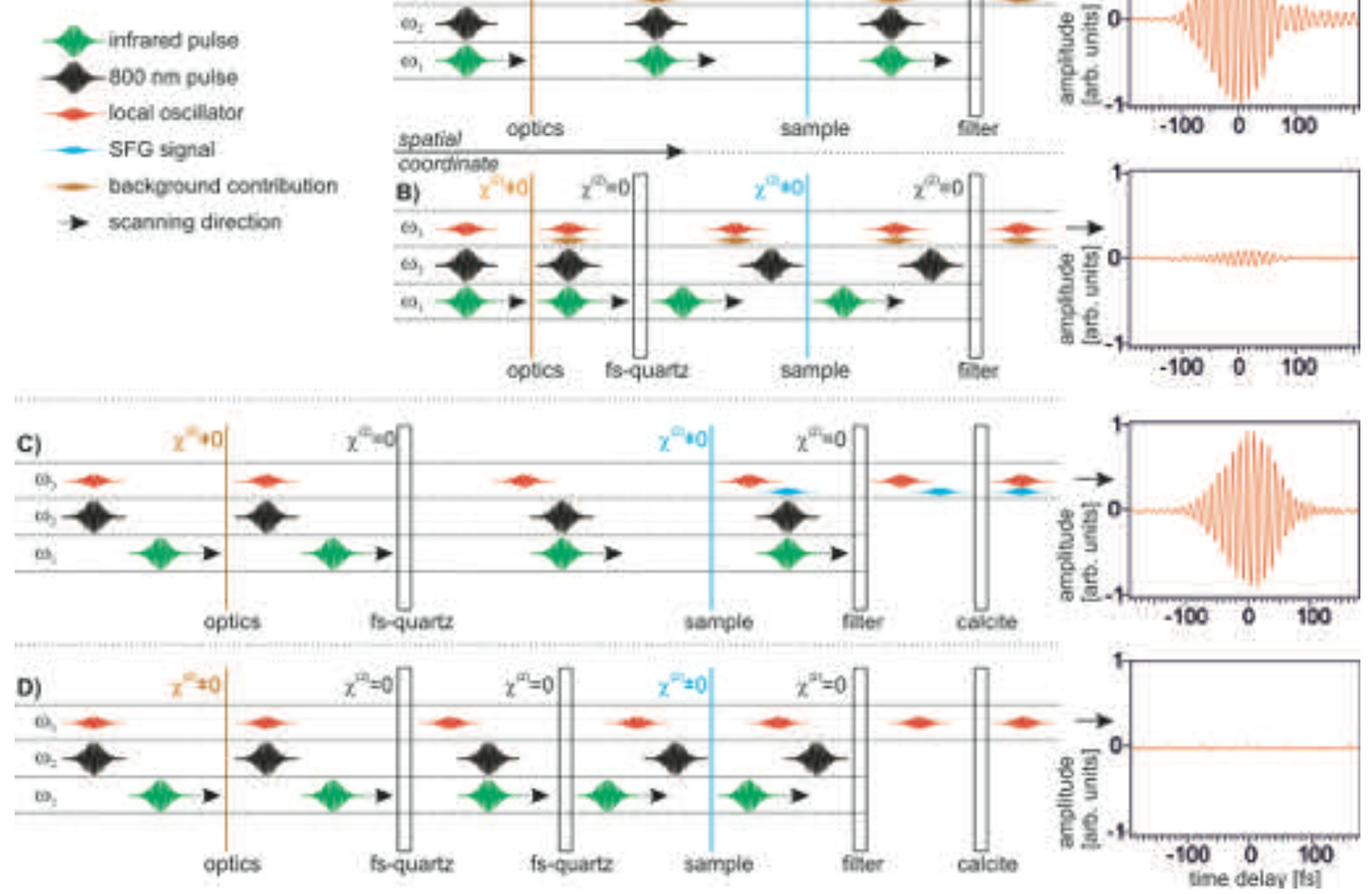

Figure 4: Analysis and suppression of background SFG contributions. A) common interferogram as obtained in our collinear setup, acquired under $p p p$ polarization combination. Introduction of a quartz plate before the sample in B) suppresses the generation of sample signal. The residual SFG originates from SFG background. In C) the pump pulses are delayed with respect to each other to exclusively overlap in time at the sample position. The resulting timing mismatch between the LO and the sample signal is compensated by inserting and adjusting the calcite crystal. D) Control experiment. A second fs quartz plate is introduced into the beam which eliminates the sample signal. For comparability all interferograms where normalized to the one shown in A).

and a background SFG contribution of unknown magnitude. Without employing a suitable technique these contributions are inseparable.

One possible way of distinguishing these signals in a collinear approach is to avoid that the pulses simultaneously overlap in time at the sample and the beam guiding optics. This can be done by introducing a $3 \mathrm{~mm}$ thick fused silica plate at normal incidence into the beam path right before the sample (see figure $4 \mathrm{~B}$ ). Due to group velocity dispersion (GVD) inside the material the different pulses get temporally separated and no longer arrive at the sample simultaneously. This temporal separation of the two pump pulses leads to a suppression of the sample signal. However, the background SFG and LO experience the same delay through GVD inside the fused silica and still overlap in time at the detector and thus show an interference signal. Note that the calcite delay crystal is not installed in that cases of figure $4 \mathrm{~A}$ and $\mathrm{B}$. The resulting interferogram is shown in figure $4 \mathrm{~B}$ and displays the isolated SFG background contribution. As expected, its amplitude is smaller than the nonlinear sample signal, but 
with a ratio of 1:10 it can easily be resolved. Considering that our sample signal originates from the relatively strong off-resonant bulk response of alpha-quartz the obtained size of the background contribution is indeed significant. For studies of samples which yield smaller nonlinear signals the relative size of the background contribution would be increased up to the point that it could even dominate the overall signal. This finding is remarkable since we took extensive precautions to avoid the generation of such signals during the design process of the spectrometer. Such precautions include e.g. a careful choice of the materials used for the installed optics and keeping reflection angles close to normal incidence. The presence of such background signals is therefore likely to be a common phenomenon in collinear spectrometers. In fact, phase shifts and spectral distortions originating from the interference of the sample signal with such background contributions may have contributed to the difficulties to obtain reproducible phase resolved SFG spectra from the air-water interface which have resulted in controversial discussions. ${ }^{14,30-32}$ It might therefore be appropriate to revisit some phase resolved SFG studies while accounting for background signals.

Using the technique presented in figure $4 \mathrm{~B}$ we can determine the amplitude and phase of the background SFG and we could in principle subtract the resulting interferogram from the total interferogram acquired before (figure $4 \mathrm{~A}$. However, it would be more convenient and certainly more accurate if the background contribution was suppressed during the sample measurement. To achieve this, we keep the previously installed fused silica plate in the beam path but move the relative pulse delays between the IR and upconversion beam such that they exclusively overlap in time at the sample position as shown in figure $4 \mathrm{C}$. Analogous to the suppression of the sample signal in figure $4 \mathrm{~B}$ the result should here be a suppression of the background contribution. However, due to the GVD in the fused silica plate the LO now lags behind the upconversion beam and hence ultimately behind the sample signal, which largely diminishes the interference amplitude. As shown in the concept section this timing mismatch can be compensated by inserting and tuning the calcite plate in the detection beam path. The resulting interferogram is depicted in figure $4 \mathrm{C}$ and shows the desired pure sample spectrum. The amplitude of the interferogram is comparable to the measurement in figure $4 \mathrm{~A}$. This confirms the successful delay compensation with the calcite plate.

The analysis of the three interferograms in figure $4 \mathrm{~A}-\mathrm{C}$ is based on the assumption that the signal suppression by introducing the respective time delays is efficient and that there is no signal generated inside the fused silica window. While the latter assumption is clearly supported by theoretical considerations (see supporting information) the validity of both assumptions can be tested experimentally. In this control experiment a second fused silica window is introduced into the beam path before the sample and the settings for the initial relative pulse delays are kept the same as in figure $4 \mathrm{C}$. The schematic representation of this experiment is shown in figure $4 \mathrm{D}$. In this configuration the background and the sample SFG should both be suppressed and according to the second assumption we do not expect any nonlinear signal from the second fused silica plate. We should thus not detect any interference signal in the experiment. The result of the measurement shown in figure $4 \mathrm{D}$ is in perfect agreement with this prediction. This confirms the validity of our analysis.

The experimental settings in figure $4 \mathrm{C}$ are thus an approach to obtain precise and background free SFG spectra within our spectrometer type while benefiting from its collinear design. Similar timing schemes can also be adopted for other experimental approaches such as frequency domain techniques in a straightforward manner. 


\section{Phase resolved SFG measure- ments of electrochemical interfaces in a liquid cell}

In the second application example we turn to the field of SFG studies of buried interfaces such as the electrode surface in an electrochemical cell. To gain insight into fundamental electrochemical processes that take place at the electrode-electrolyte interface those surfaces have been extensively characterized using homodyne vibrational SFG. ${ }^{33-36}$ The typical goal in these studies is to identify molecular species at the interface by analyzing their vibrational resonances in a SFG spectrum. One challenge in such investigations is the presence of a nonresonant (with respect to the infrared frequencies) SFG contribution $\chi_{N R}^{(2)}$ which arises from the surface of most metal electrodes ${ }^{11,37}$ and mixes with the resonant molecular responses $\chi_{R}^{(2)}$ (quite analogous to the background SFG in the previous example). The effective second order susceptibility is then given by equation 11

$$
\chi^{(2)}=\chi_{N R}^{(2)}+\chi_{R}^{(2)}
$$

Homodyned SFG techniques directly measure the spectral intensity of the emitted SFG signal: the squared modulus of the effective susceptibility. The obtained spectrum is then the frequency dependent $\left|\chi^{(2)}\right|^{2}$.

$$
\left|\chi^{(2)}\right|^{2}=\left|\chi_{N R}^{(2)}\right|^{2}+\left|\chi_{R}^{(2)}\right|^{2}+2\left|\chi_{N R}^{(2)} \chi_{R}^{(2)}\right|
$$

Problematic for the analysis of such spectra is the presence of the interference cross term $\left|\chi_{N R}^{(2)} \chi_{R}^{(2)}\right|$ which can significantly alter the spectral shape of the resonant features in a homodyned spectrum, depending on the phase and amplitude of the non-resonant contribution. $\chi_{N R}^{(2)}$, however, exhibits an often unknown spectral phase and amplitude, which has been shown to sensitively depend on the surface potential and surface electronic structure. ${ }^{38}$ For potential dependent measurements it is therefore highly non-trivial to evaluate whether observed changes in a measured homodyned SFG spectrum originate from changes in the resonant response of the interfacial molecular species caused by e.g. changes in molecular orientation or structural changes as a result of electrochemical reactions or from a change in the non-resonant response. Obtaining a correct interpretation of the observed spectral changes is without further knowledge hardly possible. Applying heterodyned vibrational SFG spectroscopy to these systems can overcome this ambiguity because the quantity $\chi^{(2)}$ is directly measured as in equation 11. This allows for separately analyzing changes in the two contributions. However, to our knowledge such measurements have so far not been realized. ${ }^{11}$

Performing heterodyned SFG spectroscopy on these buried liquid-solid interfaces is a very similar experimental to that shown in figure $4 \mathrm{C}$. To prevent the evaporation of the solvent such cells are typically covered by some window material (see figure 5A) Since all beams must pass through the window and the liquid to access the interface of interest they experience a GVD similar to the case of introducing the fused silica plate into the beams. As for the fused silica case the resulting timing mismatch between LO and SFG signal can be compensated by tuning the calcite plate in the detection beam path.

To demonstrate the functionality of our approach for the investigation of electrochemical interfaces we present SFG measurements of a self-assembled monolayer (SAM) of octadecanethiol (ODT) on a gold mirror. The sum frequency spectrum of the Au-ODT SAM in air is dominated in the $\mathrm{C}-\mathrm{H}$ stretch region by three distinct resonance peaks from the terminal methyl groups of the molecular chain. ${ }^{39}$ These resonant peaks can be attributed to the symmetric $\mathrm{C}-\mathrm{H}$-stretch vibrations at $\nu \approx$ $2875 \mathrm{~cm}^{-1}$ and $\nu \approx 2935 \mathrm{~cm}^{-1}$ (Fermi resonance) and to the anti-symmetric stretch vibration at $\nu \approx 2962 \mathrm{~cm}^{-1}$. ${ }^{39}$ Two additional small peaks usually observed in ODT SAMs at $\nu \approx 2850 \mathrm{~cm}^{-1}$ and $\nu \approx 2900 \mathrm{~cm}^{-1}$ are related to gauche defects in the SAM. ${ }^{39}$

To mimic the experimental situation in an electrochemical cell we enclosed the ODT covered gold surface in a liquid cell (see figure 5A). 
This cell consists of the sample and a $50 \mu \mathrm{m}$ thin Teflon spacer covered with a calciumfluoride window. The small volume between the sample and the window is filled with deuterated acetonitrile.

In a first measurement the sum frequency spectrum of the sample is acquired without compensating for the GVD in the $\mathrm{CaF}_{2} 2$ window followed by the same experiment with the calcite crystal installed in the detection beam path for compensation. The resulting magnitude spectra are depicted in figure 5B. The spectrum obtained with the GVD compensation (black line) nicely shows the three expected resonance peaks (appearing as dips) of the ODT SAM on top of a broad non-resonant background signal which originates from the Au surface. The spectrum obtained without compensation (green line) in contrast, shows a largely reduced signal intensity which is only slightly above the noise level in the spectrum. Interestingly, the relative phases between resonant and non-resonant contributions seem here to be inverted. This result clearly shows that the timing mismatch between the LO and the SFG signal leads to severe spectral distortions and confirms the necessity of the time delay compensation.

In order to verify if our delay compensation technique yields an accurate spectrum we also compare the result to a spectrum of the AuODT sample exposed to air where no compensation is required (see figure $5 \mathrm{C}$ ).

The well ordered and dense structure of the SAM should, for steric reasons, prevent the molecules from changing their orientation or structure when in contact with the liquid. We thus expect that the nonlinear spectral responses of the ODT monolayer are similar in both experiments. Figure 5C shows the imaginary (absorptive) part of the second order susceptibility for the Au-ODT sample which are extracted from the measurements in the liquid cell (black line) and in air (red dotted line), respectively. Comparing the line shapes of the three resonance peaks from the ODT layer shows indeed a very close match between the two spectra. The most prominent differences appear in the spectrum from the liquid cell in regions outside the frequency window of the

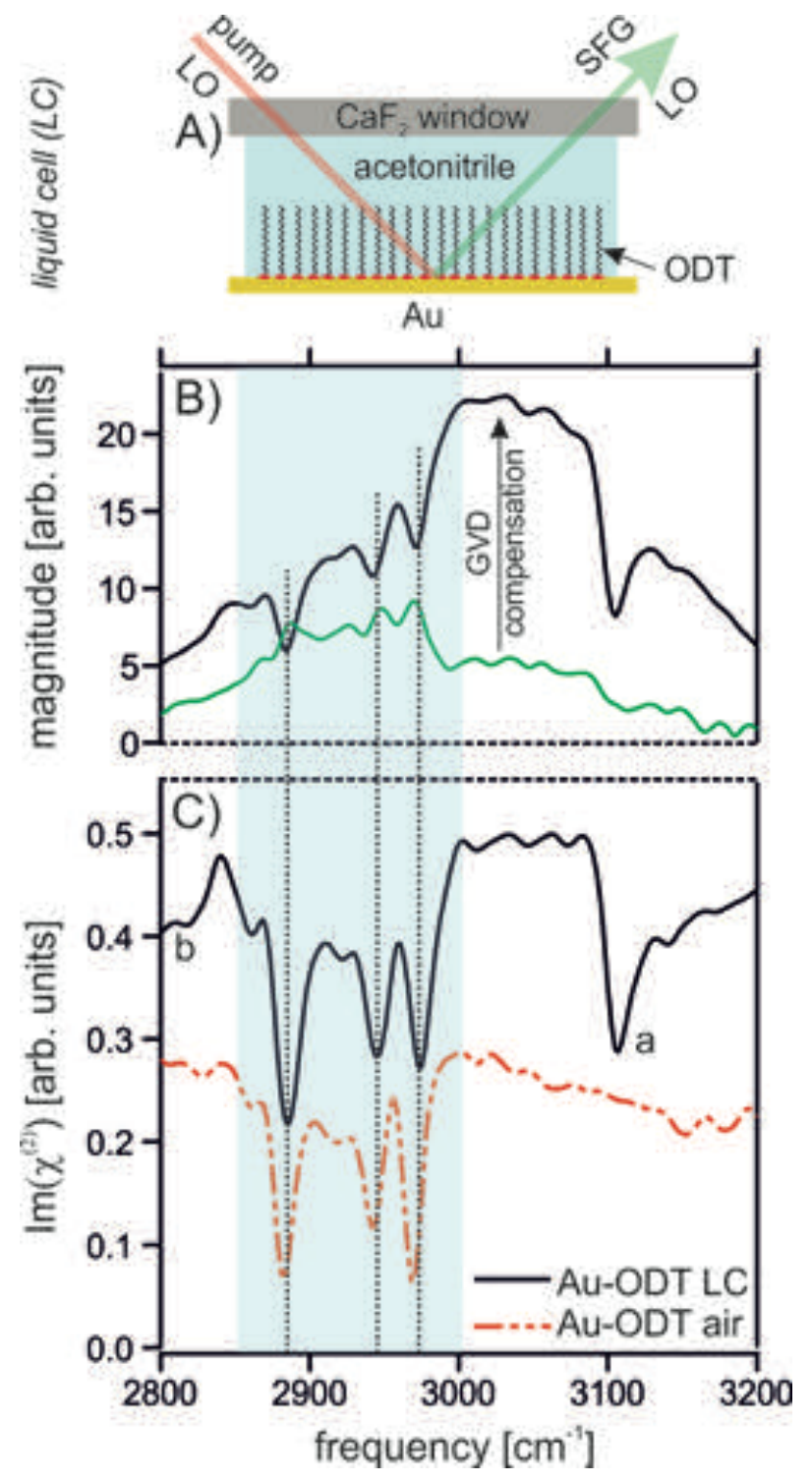

Figure 5: Phase resolved SFG measurements of Au-ODT in a liquid cell acquired under $p p p$ polarization combination: A) sketch of the experimental configuration, B) raw magnitude SFG spectra of Au-ODT under acetonitrile with (black line) and without GVD compensation (green line). C) Imaginary parts of $\chi(2)$ for $\mathrm{Au}-\mathrm{ODT}$ under acetonitrile (black line) and in air (red dotted line, vertically shifted for better visibility). The spectra in $\mathrm{C}$ ) are corrected for the spectral profile of the IR pulse. Dotted black lines indicate peak positions from the ODT response.

ODT resonances. They are labeled $\mathrm{a}$ and $\mathrm{b}$ (onset of a negative peak) in figure $5 \mathrm{C}$. Since these peaks also appear in a control experiment of the 
same cell without ODT (not shown in this contribution) they must originate from molecular species which are introduced with the solvent. However, deuterated acetonitrile does not show significant bulk absorption peaks in the measured region. We therefore, conclude that the observed peaks stem from the solvent related formation of interfacial species which interact with the Au layer. While a more detailed investigation of the origin of peaks a and $b$ is beyond the scope of this contributions the comparison to the Au-ODT spectrum in air indicates that the phase resolved spectrum measured in the liquid cell is indeed accurate.

Furthermore, this example shows more generally how our timing control technique enables us to extent the capabilities of our collinear spectrometer e.g. to SFG studies on electrodes in electrochemical cells.

\section{Summary}

We have presented an experimental method that allows for precise timing control between the local oscillator and the nonlinear signal in collinear spectrometers for second order nonlinear spectroscopy. By exploiting the polarization dependent group delay in a birefringent crystal in combination with a polarization based balanced detection scheme this technique yields continuous tunability of the relative pulse delay without imposing any particular requirements on the polarizations of pump pulses or the detected sample signal. The presented solution for the timing control is very general and can readily be implemented in most of the common spectrometer types including time domain and frequency domain approaches. That way it is possible to benefit from the superior phase accuracy and a simplified spectrometer alignment of a collinear design while maintaining full experimental flexibility.

The applicability of this technique goes beyond simple measurements of nonlinear spectra in a collinear spectrometer. As demonstrated in the result section we can use the ability to control the pulse delay to freely modify the pulse sequences in different places inside the spec- trometer. We have shown that this can be used to either isolate or suppress the nonlinear background contributions which originate from the beam steering optics inside the optical setup. Our results indicate that such background signals can indeed have a significant size in collinear setups which potentially results in falsified nonlinear sample spectra. The presented suppression method is therefore an essential ingredient to ensure high accuracy in the nonlinear spectra obtained with a collinear approach.

Furthermore we have demonstrated how the technique extends the capabilities of our time domain SFG spectrometer to measure phase resolved spectra of buried interfaces which are enclosed in a liquid cell. These types of measurements will be further pursued to investigate the fundamental processes at electrochemical interfaces.

Acknowledgement This study was supported by the European Research Council (ERC) under the European Union's Horizon 2020 research and innovation program (Grant No. 772286 to R.K.C.).

Supporting Information Available: This material is available free of charge via the Internet at http://pubs.acs.org/.

\section{References}

(1) Fu, L.; Wang, Z.; Batista, V. S.; Yan, E. C. New Insights from Sum Frequency Generation Vibrational Spectroscopy into the Interactions of Islet Amyloid Polypeptides with Lipid Membranes. Journal of diabetes research 2015, 2016.

(2) Fu, L.; Wang, Z.; Yan, E. C. Chiral Vibrational Structures of Proteins at Interfaces Probed by Sum Frequency Generation Spectroscopy. International Journal of Molecular Sciences 2011, 12, 94049425.

(3) Zwaschka, G.; Wolf, M.; Campen, R. K.; Tong, Y. A Microscopic Model of the Electrochemical Vibrational Stark Ef- 
fect: Understanding VSF Spectroscopy of (bi)Sulfate on $\operatorname{Pt}(111)$. Surface Science 2018, 678, 78-85.

(4) Jubb, A. M.; Hua, W.; Allen, H. C. Organization of Water and Atmospherically Relevant Ions and Solutes: Vibrational Sum Frequency Spectroscopy at the Vapor/Liquid and Liquid/Solid Interfaces. Accounts of Chemical Research 2012, 45, 110-119.

(5) Humbert, C.; Noblet, T.; Dalstein, L.; Busson, B.; Barbillon, G. Sum-Frequency Generation Spectroscopy of Plasmonic Nanomaterials: A Review. Materials 2019, 12, 836.

(6) Tegeder, P. Optically and thermally induced molecular switching processes at metal surfaces. Journal of Physics: Condensed Matter 2012, 24, 394001.

(7) Stiopkin, I. V.; Jayathilake, H. D.; Bordenyuk, A. N.; Benderskii, A. V. Heterodyne-Detected Vibrational Sum Frequency Generation Spectroscopy. Journal of the American Chemical Society 2008, 130, 2271-2275.

(8) Shen, Y. R. Surfaces probed by nonlinear optics. Surface Science 1994, 299, 551562.

(9) Lambert, A. G.; Davies, P. B.; Neivandt, D. J. Implementing the Theory of Sum Frequency Generation Vibrational Spectroscopy: A Tutorial Review. Applied Spectroscopy Reviews 2005, 40, 103-145.

(10) Nihonyanagi, S.; Yamaguchi, S.; Tahara, T. Direct evidence for orientational flip-flop of water molecules at charged interfaces: A heterodyne-detected vibrational sum frequency generation study. The Journal of Chemical Physics 2009, 130, 204704.

(11) Shen, Y. R. Fundamentals of SumFrequency Spectroscopy; Cambridge University Press, 2016.
(12) Pettinger, B.; Bilger, C. A novel approach to analyze the optical second harmonic generation anisotropy at surfaces employing interference techniques. Example: the $\mathrm{Au}(110)$ electrode. Chemical Physics Letters 1998, 286, 355-360.

(13) Laaser, J. E.; Xiong, W.; Zanni, M. T. Time-Domain SFG Spectroscopy Using Mid-IR Pulse Shaping: Practical and Intrinsic Advantages. The Journal of Physical Chemistry B 2011, 115, 2536-2546.

(14) Sun, S.; Liang, R.; Xu, X.; Zhu, H.; Shen, Y. R.; Tian, C. Phase reference in phase-sensitive sum-frequency vibrational spectroscopy. The Journal of Chemical Physics 2016, 144, 244711.

(15) Xu, B.; Wu, Y.; Sun, D.; Dai, H.-L.; Rao, Y. Stabilized phase detection of heterodyne sum frequency generation for interfacial studies. Optics Letters 2015, 40, 4472 .

(16) Vanselous, H.; Petersen, P. B. Extending the Capabilities of Heterodyne-Detected Sum-Frequency Generation Spectroscopy: Probing Any Interface in Any Polarization Combination. The Journal of Physical Chemistry C 2016, 120, 8175-8184.

(17) Thämer, M.; Campen, R. K.; Wolf, M. Detecting weak signals from interfaces by high accuracy phase-resolved SFG spectroscopy. Physical Chemistry Chemical Physics 2018, 20, 25875-25882.

(18) Nihonyanagi, S.; Mondal, J. A.; Yamaguchi, S.; Tahara, T. Structure and Dynamics of Interfacial Water Studied by Heterodyne-Detected Vibrational SumFrequency Generation. Annual Review of Physical Chemistry 2013, 64, 579-603.

(19) Shen, Y. Phase-Sensitive Sum-Frequency Spectroscopy. Annual Review of Physical Chemistry 2013, 64, 129-150.

(20) Richter, L. J.; Petralli-Mallow, T. P.; Stephenson, J. C. Vibrationally resolved 
sum-frequency generation with broadbandwidth infrared pulses. Optics letters 1998, 23, 1594-1596.

(21) Thämer, M.; Garling, T.; Campen, R. K.; Wolf, M. Quantitative determination of the nonlinear bulk and surface response from alpha-quartz using phase sensitive SFG spectroscopy. The Journal of Chemical Physics 2019, 151, 064707.

(22) Yamaguchi, S.; Tahara, T. Heterodynedetected electronic sum frequency generation: "Up" versus "down" alignment of interfacial molecules. The Journal of Chemical Physics 2008, 129, 101102.

(23) Ji, N.; Ostroverkhov, V.; Chen, C.Y.; Shen, Y.-R. Phase-Sensitive SumFrequency Vibrational Spectroscopy and Its Application to Studies of Interfacial Alkyl Chains. Journal of the American Chemical Society 2007, 129, 1005610057.

(24) Wang, H.; Gao, T.; Xiong, W. Self-PhaseStabilized Heterodyne Vibrational Sum Frequency Generation Microscopy. ACS Photonics 2017, 4, 1839-1845.

(25) Wilson, J. W.; Bartels, R. A. Rapid Birefringent Delay Scanning for Coherent Multiphoton Impulsive Raman Pump-Probe Spectroscopy. IEEE Journal of Selected Topics in Quantum Electronics 2012, 18, 130-139.

(26) Guenther, B. D. Modern Optics; Oxford University Press, 2015.

(27) Schlup, P.; Wilson, J.; Hartinger, K.; Bartels, R. A. Dispersion balancing of variable-delay monolithic pulse splitters. Applied optics 2007, 46, 5967-5973.

(28) Jones, K. C.; Ganim, Z.; Tokmakoff, A. Heterodyne-Detected Dispersed Vibrational Echo Spectroscopy. The Journal of Physical Chemistry A 2009, 113, 1406014066.
(29) Ohno, P. E.; Chang, H.; Spencer, A. P.; Liu, Y.; Boamah, M. D.; Wang, H.-f.; Geiger, F. M. Beyond the Gouy-Chapman Model with Heterodyne-Detected Second Harmonic Generation. The Journal of Physical Chemistry Letters 2019, 10, 2328-2334.

(30) Nihonyanagi, S.; Ishiyama, T.; Lee, T.k.; Yamaguchi, S.; Bonn, M.; Morita, A.; Tahara, T. Unified Molecular View of the Air/Water Interface Based on Experimental and Theoretical $\mathrm{x}{ }^{(2)}$ Spectra of an Isotopically Diluted Water Surface. Journal of the American Chemical Society 2011, 133, 16875-16880.

(31) Nihonyanagi, S.; Kusaka, Ryoji,; Inoue, Ken-Ichi,; Adhikari, Aniruddha,; Yamaguchi, Shoichi,; Tahara, Tahei, Accurate determination of complex $x{ }^{(2)}$ spectrum of the air/water interface. The Journal of Chemical Physics 2015, 143, 124707.

(32) Tian, C.-S.; Shen, Y. R. Isotopic Dilution Study of the Water/Vapor Interface by Phase-Sensitive Sum-Frequency Vibrational Spectroscopy. Journal of the American Chemical Society 2009, 131, 27902791.

(33) Tong, Y.; Lapointe, F.; Thämer, M.; Wolf, M.; Campen, R. K. Hydrophobic Water Probed Experimentally at the Gold Electrode/Aqueous Interface. Angewandte Chemie International Edition 2017, 56, 4211-4214.

(34) Baldelli, S.; Markovic, N.; Ross, P.; Shen, Y.-R.; Somorjai, G. Sum Frequency Generation of CO on (111) and Polycrystalline Platinum Electrode Surfaces: Evidence for SFG Invisible Surface CO. The Journal of Physical Chemistry B 1999, 103, 8920-8925.

(35) Wang, J.; Xu, M.; Huangfu, Z.; Wang, Y.; He, Y.; Guo, W.; Wang, Z. Observation of gold electrode surface response to the adsorption and oxidation of thiocyanate 
in acidic electrolyte with broadband sumfrequency generation spectroscopy. Vibrational Spectroscopy 2016, 85, 122-127.

(36) Tadjeddine, A.; Guyot-Sionnest, P. Spectroscopic investigation of adsorbed cyanide and thiocyanate on platinum using sum frequency generation. Electrochimica acta 1991, 36, 1849-1854.

(37) Vidal, F.; Tadjeddine, A. Sum-frequency generation spectroscopy of interfaces. Reports on Progress in Physics 2005, 68, 1095-1127.

(38) Bilger, C.; Pettinger, B. Potential dependence of the non-linear polarizability of an $\mathrm{Au}$ (110) electrode. Journal of the Chemical Society, Faraday Transactions 1998, 94, 2795-2801.

(39) Potterton, E.; Bain, C. Infrared-infrared sum-frequency generation from adsorbates on metal surfaces. Journal of Electroanalytical Chemistry 1996, 409, 109-114. 
Graphical TOC Entry

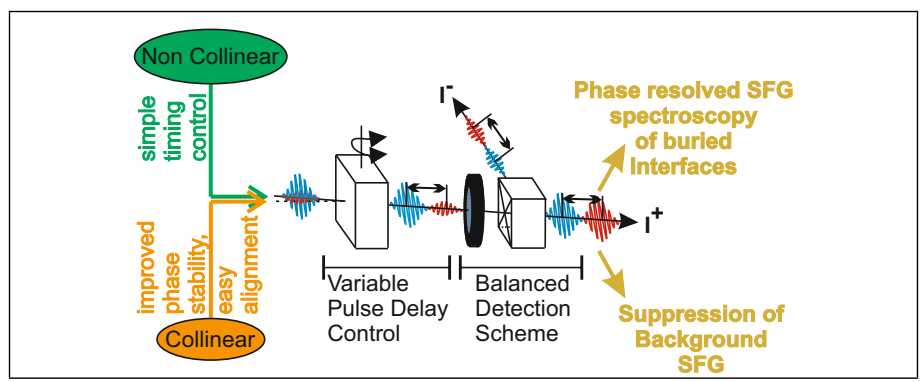

